\title{
Sparse Approximations for High Fidelity Compression of Network Traffic Data
}

\author{
William Aiello $^{\dagger}$ \\ University of British Columbia \\ aiello@cs.ubc.ca
}

\author{
Anna Gilbert ${ }^{\S}$ \\ University of Michigan \\ annacg@umich.edu
}

\author{
Vyas Sekar $¥$ \\ Carnegie Mellon University \\ vyass@cs.cmu.edu
}

\author{
Brian Rexroad \\ $A T \& T$ Labs \\ brexroad@att.com
}

\begin{abstract}
An important component of traffic analysis and network monitoring is the ability to correlate events across multiple data streams, from different sources and from different time periods. Storing such a large amount of data for visualizing traffic trends and for building prediction models of "normal" network traffic represents a great challenge because the data sets are enormous. In this paper we present the application and analysis of signal processing techniques for effective practical compression of network traffic data. We propose to use a sparse approximation of the network traffic data over a rich collection of natural building blocks, with several natural dictionaries drawn from the networking community's experience with traffic data. We observe that with such natural dictionaries, high fidelity compression of the original traffic data can be achieved such that even with a compression ratio of around 1:6, the compression error, in terms of the energy of the original signal lost, is less than $1 \%$. We also observe that the sparse representations are stable over time, and that the stable components correspond to well-defined periodicities in network traffic.
\end{abstract}

\section{Introduction}

Traffic monitoring is not a simple task. Network operators have to deal with large volumes of data, and need to identify and respond to network incidents in real-time. The task is complicated even further by the fact that monitoring needs to be done on multiple dimensions and timescales. It is evident that network operators wish to observe traffic at finer granularities across different dimensions for a multitude of reasons that include: 1 . real-time detection and

\footnotetext{
$\dagger$ Majority of this work was done when the author was a member of AT \& T Labs-Research

${ }^{\ddagger}$ Majority of this work was done when the author was a research intern at AT \& T Labs-Research

$\S$ Majority of this work was done when the author was a member of AT \& T Labs-Research. The author is supported by an Elizabeth Crosby Faculty Fellowship.
}

response to network failures and isolating errant network segments, 2. real-time detection of network attacks such as DDoS and worms, and installation of filters to protect network entities, and 3. finer resolution root-cause analysis of the incidents and automated/semi-automated drill down of the incident.

To meet these requirements, we must be able to generate and store traffic data on multiple resolution scales in space (network prefixes and physical network entities such as links, routers), and in time (storing the traffic aggregates at multiple time resolutions). Such requirements naturally translate into increased operational costs due to the increased storage requirement. We often transport large portions of the historical data across a network to individual operators, import pieces of data into statistical analysis and visualization software for modeling purposes, and index and run queries against various historical databases for data drill down. Thus the management overhead involved in handling such large data sets, and the computational overhead in accessing and processing the large volumes of historical data also increases. We must reduce the storage size of the data, not only for efficient management of historical traffic data, but also to accommodate fine data resolution across space and time.

The compression techniques we investigate are "lossy" compression methods. For most network monitoring applications that utilize historical traffic data, it often suffices to capture salient features of the underlying traffic. We can thus afford some error by ignoring the low-energy stochastic components of the signal, and gain better compression using lossy compression techniques (as opposed to lossless compression methods such as gzip [11] which reduce the storage size of the data only and do not reduce the size of the input to monitoring applications). The overall goal of such compression techniques is to obtain high fidelity (i.e. low error) representations with as little storage as possible.

In particular, we use a compression method called sparse representation over redundant dictionaries. A visual inspection of aggregated network traffic for many high vol- 
ume ports reveals three components. First, there is a natural diurnal variation for many ports and/or other periodic variations as well. Second, there are spikes, dips, and other components of the traffic that appear to be the result of non-periodic events or processes. Finally, the traffic appears to be stochastic over small time scales with variance much smaller than the periodic variations for high volume ports. Representing a signal with all three components using a single orthonormal basis, such as a Fourier basis or a wavelet representation is not likely to yield good compression: a basis that represents periodic signals well will not represent non-periodic signals efficiently and vice versa. The methods presented in this paper allow us to use two or more orthonormal bases simultaneously. A set of two or more orthonormal bases is called a redundant dictionary. Hence, with an appropriate set of orthonormal bases as the redundant dictionary, the periodic and the significant nonperiodic portions of the traffic time series can both be represented efficiently within the same framework.

Sparse representation or approximation over redundant dictionaries does not make assumptions about the underlying distributions in the traffic time series. As a result, sparse approximation can guarantee high fidelity regardless of changes in the underlying distributions. In addition, there are highly efficient, provably correct algorithms for solving sparse approximation problems. These algorithms scale with the data and can be easily adapted to multiple sources of data. They are greedy algorithms, known as matching or orthogonal matching pursuit.

The primary contribution of this paper is a rigorous investigation of the method of sparse representation over redundant dictionaries for the compression of network time series data. We propose and evaluate several redundant dictionaries that are naturally suited for traffic time series data. We conclude that these methods achieve significant compression with very high fidelity across a wide spectrum of traffic data. In addition, we also observe that the sparse representations are stable, not only in terms of their selection in the sparse representation over time but also in terms of the individual amplitudes in the representation. These stable components correspond to well-defined periodicities in network traffic, and capture the natural structure of traffic time series data. To the best of our knowledge, this is the first thorough application of sparse representations for compressing network traffic data.

We discuss related work in Section 2, and present a overall motivation for compression in Section 3. In Section 4 we describe in more detail the framework of matching (greedy) pursuit over redundant dictionaries. Section 5 describes our traffic data set, derived from a large Internet provider. We evaluate the efficacy of our compression techniques in Section 6. Section 7 presents some network traffic monitoring applications that demonstrate the utility of the compression methods we used. Section 8 discusses the scope for improving the compression, before we conclude in Section 9.

\section{Related Work}

Statisticians concern themselves with subset selection in regression [13] and electrical engineers use sparse representations for the compression and analysis of audio, image, and video signals (see $[4,6,12]$ for several example references).

Lakhina, et al. [9, 10] examine the structure of network traffic using Principal Component Analysis (PCA). The observations in our work provide similar insight into the structure of network traffic. There are two compelling reasons for using sparse approximations over redundant dictionaries, as opposed to PCA alone, for obtaining similar fidelity-compression tradeoffs. First, the description length for sparse approximation is much shorter than for PCA, since the principal vectors require substantially more space to represent than simple indices into a dictionary. Second, PCA like techniques may capture and identify the (predominant) structure across all measurements, but may not be adequate for representing subtle characteristics on individual traffic aggregates.

Barford, et al. [1] use pseudo-spline wavelets as the basis wavelet to analyze the time localized normalized variance of the high frequency component to identify signal anomalies. The primary difference is our application of signal processing techniques for compressing network traffic data, as opposed to using signal decomposition techniques for isolating anomalies in time series data.

There are several methods for data reduction for generating compact traffic summaries for specific real-time applications. Sketch based methods [8] have been used for anomaly detection on traffic data, while Estan et al. [3] discuss methods for performing multi-dimensional analysis of network traffic data. While such approaches are appealing for real-time traffic analysis with low CPU and memory requirements, they do not address the problems of dealing with large volumes of historical data that arise in network operations. A third, important method of reducing data is sampling [2] the raw data before storing historical information. However, in order for the sampled data to be an accurate reflection of the raw data, one must make assumptions regarding the underlying traffic distributions.

\section{Compression}

It is easy to (falsely) argue that compression techniques have considerably less relevance when the current cost of (secondary) storage is less than \$1 per GB. Large operational networks indeed have the unenviable task of managing many terabytes of measurement data on an ongoing 
basis, with multiple data streams coming from different routers, customer links, and measurement probes. While it may indeed be feasible to collect, store, and manage such a large volume of data for small periods of time (e.g. for the last few days), the real problem is in managing large volumes of historical data. Having access to historical data is a crucial part of a network operator's diagnostic toolkit. The historical datasets are typically used for building prediction models for anomaly detection, and also for building visual diagnostic aids for network operators. The storage requirement increases not only because of the need for access to large volumes of historical traffic data, but also the pressing need for storing such historical data across different spatial and temporal resolutions, as reference models for fine-grained online analysis.

It may be possible to specify compression and summarization methods for reducing the storage requirement for specific traffic monitoring applications that use historical data. However, there is a definite need for historical reference data to be stored at fine spatial and temporal resolutions for a wide variety of applications, and it is often difficult to ascertain the set of applications and diagnostic techniques that would use these datasets ahead of time. The compression techniques discussed in this paper have the desirable property that they operate in an application-agnostic setting, without making significant assumptions regarding the underlying traffic distributions. Since many traffic monitoring applications can tolerate a small amount of error in the stored values, lossy compression techniques that can guarantee a high fidelity representation with small storage overhead are ideally suited for our requirements. We find that our techniques provide very accurate compressed representations so that there is only a negligible loss of accuracy across a wide spectrum of traffic monitoring applications.

The basic idea behind the compression techniques used in this paper is to obtain a sparse representation of the given time series signal using different orthonormal and redundant bases. While a perfect lossless representation can be obtained by keeping all the coefficients of the representation (e.g. using all Fourier or wavelet coefficients), we can obtain a compressed (albeit lossy) representation by only storing the high energy coefficients, that capture a substantial part of the original time series signal.

Suppose we have a given time series signal of length $N$. For example, in our data set consisting of hourly aggregates of traffic volumes, $\mathrm{N}=168$ over a week, for a single traffic metric of interest. We can obtain a lossless representation by using up a total storage of $N \times k$ bits, where $k$ represents the cost of storing each data point. Alternatively, we can obtain a sparse representation using $m$ coefficients using a total storage space of $m \times k^{\prime}+|D|$ bits, where the term $|D|$ represents the length of the dictionary used for compression, and $k^{\prime}$ represents the cost of storing the amplitude associated with each coefficient. The $|D|$ term represents the cost of storing the list of selected indices as a bit-vector of length equal to the size of the dictionary. The length of the dictionary $|D|$ is equal to $\alpha N$, with the value $\alpha$ being one for an orthonormal basis (e.g., Fourier, Wavelet, Spike) or equal to two in the case of a redundant dictionary consisting of Fourier and Spike waveforms. The effective compression ratio is thus $\left(m k^{\prime}+\alpha N\right) /(N k)$. Assuming $k \approx k^{\prime}$ (the cost of storing the raw and compressed coefficients are similar) and $\alpha \ll k$ (the values in consideration are large integers or floats), the effective compression (even with this naive encoding) is approximately equal to $m / N$ ${ }^{1}$. The primary focus of this paper is not to come up with an optimal encoding scheme for storing the $m$ coefficients to extract the greatest per-bit compression. Rather we wish to explore the spectrum of signal compression techniques, using different natural waveforms as dictionaries for achieving a reasonable error-compression tradeoff.

A natural error metric for lossy compression techniques in signal processing is the energy of the residual, which is the vector difference between the original signal and the compressed representation. Let $S$ be the original signal and $C_{s}$ represent the compressed representation of $S$. The signal $R=S-C_{s}$ represents the residual signal. We use the following relative error metric $\frac{\|R\|^{2}}{\|S\|^{2}}$ where $\|\cdot\|$ represents the $L_{2}$ (Euclidean) norm of a vector. The error metric represents the fraction of the energy in the original signal that is not captured in the compressed model. For example, a relative error of 0.01 implies that the energy of the residual signal (not captured by the compressed representation) is only $1 \%$ of the energy of the original signal. Our results indicate that we can achieve high fidelity compression for more than $90 \%$ of all traffic aggregates, with a relative error of less than 0.01 using only $m=30$ coefficients, for the hourly aggregates with $N=168$. Since a m-coefficient representation of the signal implies a compression ratio of roughly $m / N$, with $N=168$, a 30-coefficient representation corresponds to a compression ratio of roughly 1:6.

Consider the following scenario. An operator wishes to have access to finer resolution historical reference data collected on a per application port basis (refer Section 5 for a detailed description of the datasets used in this paper). Suppose the operator wants to improve the temporal granularity by going from hourly aggregates to 10 minute aggregates. The new storage requirement is a non-negligible $60 / 10 \times X=6 X$, where $X$ represents the current storage requirement (roughly $1 \mathrm{~GB}$ of raw data per router per week). Using the compression techniques presented in this paper, by finding small number of dictionary components to represent the time series data, the operator can easily offset this increased storage cost.

Further, we observe (refer Section 8.2) that moving to finer temporal granularities does not actually incur substantially higher storage cost. For example we find that the 
same fidelity of compression (at most $1 \%$ error) can be obtained for time-series data at fine time granularity (aggregated over five minute intervals) by using a similar number of coefficients as those used for data at coarser time granularities (hourly aggregates). Thus by using our compression techniques operators may in fact be able to substantially cut down storage costs, or alternatively use the storage "gained" for improving spatial granularities (collecting data from more routers, customers, prefixes, etc).

In the next section, we present a brief overview on the use of redundant dictionaries for compression, and present a greedy algorithm for finding a sparse representation over a redundant dictionary.

\section{Sparse Representations over Redundant Dictionaries}

One mathematically rigorous method of compression is that of sparse approximation. Sparse approximation problems arise in a host of scientific, mathematical, and engineering settings and find greatest practical application in image, audio, and video compression $[4,6,12]$, to name a few. While each application calls for a slightly different problem formulation, the overall goal is to identify a good approximation involving a few elementary signalsa sparse approximation. Sparse approximation problems have two characteristics. First, the signal vector is approximated with a linear model of elementary signals (drawn from a fixed collection of several orthonormal bases). Second, there is a compromise between approximation error (usually measured with Euclidean norm) and the number of elementary signals in the linear combination.

One example of a redundant dictionary for signals of length $N$ is the union

$$
\mathcal{D}=\left\{\cos \left(\frac{\pi k\left(t+\frac{1}{2}\right)}{N}\right)\right\} \bigcup\left\{\delta_{k}(t)\right\},
$$

where $k=0, \ldots, N-1$, of the cosines and the spikes on $N$ points. The "spike" function $\delta_{k}(t)$ is zero if $t \neq k$ and is one if $t=k$. Either basis of vectors is complete enough to represent a time series of length $N$ but it might take more vectors in one basis than the other to represent the signal. To be concrete, let us take the signal

$$
X(t)=3 \cos \left(\frac{\pi 8\left(t+\frac{1}{2}\right)}{100}\right)-5 \delta_{10}(t)+15 \delta_{20}(t)
$$

plotted in Figure 1(a). The spectrum of the discrete cosine transform (DCT) of $X$ is plotted in Figure 1(b). For this example, all the coefficients are nonzero. That is, if we write

$$
X(t)=\frac{1}{100} \sum_{k=0}^{99} \hat{X}(k) \cos \left(\frac{\pi k\left(t+\frac{1}{2}\right)}{100}\right)
$$

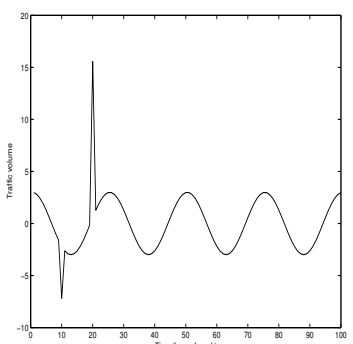

(a) An example signal $X$ which has a short representation over the redundant dictionary $\mathcal{D}$.

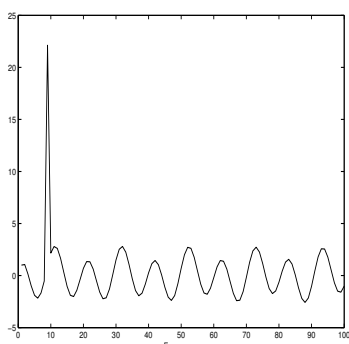

(b) The discrete cosine transform (DCT) of the example signal $X$.
Figure 1: The example signal $X$ and its discrete cosine transform (DCT).

as a linear combination of vectors from the cosine basis, then all 100 of the coefficients $\hat{X}(k)$ are nonzero. Also, if we write $X(t)$ as a linear combination of spikes, then we must use almost all 100 coefficients as the signal $X(t)$ is nonzero in almost all 100 places. Contrast these two expansions for $X(t)$ with the expansion over the redundant dictionary $\mathcal{D}$

$$
X(t)=3 \cos \left(\frac{\pi 8\left(t+\frac{1}{2}\right)}{100}\right)-5 \delta_{10}(t)+15 \delta_{20}(t) .
$$

In this expansion there are only three nonzero coefficients, the coefficient 3 attached to the cosine term and the two coefficients associated with the two spikes present in the signal. Clearly, it is more efficient to store three coefficients than all 100. With three coefficients, we can reconstruct or decompress the signal exactly. For more complicated signals, we can keep a few coefficients only and obtain a good approximation to the signal with little storage. We obtain a high fidelity (albeit lossy) compressed version of the signal. Observe that because we used a dictionary which consists of simple, natural building blocks (cosines and spikes), we need not store 100 values to represent each vector in the dictionary. We do not have to write out each cosine or spike waveform explicitly.

Finding the optimal dictionary for a given application is a difficult problem and good approximations require domain specific heuristics. Our contribution is the identification of a set of dictionaries that are well-suited for compressing traffic time-series data, and in empirically justifying the choice of such dictionaries. Prior work on understanding the dimensionality of network traffic data using principal component analysis [10] identifies three types of eigenflows: periodic, spikes, and noise. With this intuition, we try different dictionaries drawn from three basic waveforms: periodic functions (or complex exponentials), 
spikes, and wavelets. Dictionaries that are comprised of these constituent signals are descriptive enough to capture the main types of behavior but not so large that the algorithms are unwieldy.

\subsection{Greedy Pursuit Algorithms}

A greedy pursuit algorithm at each iteration makes the best local improvement to the current approximation in hope of obtaining a good overall solution. The primary algorithm is referred to as Orthogonal Matching Pursuit (OMP), described in Algorithm 4.1. In each step of the algorithm, the current best waveform is chosen from the dictionary to approximate the residual signal. That waveform is then subtracted from the residual and added to the approximation. The algorithm then iterates on the residual. At the end of the pursuit stage, the approximation consists of a linear combination of a small number of basic waveforms. We fix some notation before describing the algorithm. The dictionary $\mathcal{D}$ consists of $d$ vectors $\varphi_{j}$ of length $N$ each. We write these vectors $\varphi_{j}$ as the rows in a matrix $\Phi$ and refer to this matrix as the dictionary matrix. OMP is one of the fastest ${ }^{2}$ provably correct algorithm for sparse representation over redundant dictionaries, assuming that the dictionary satisfies certain geometric constraints [5] (roughly, the vectors in the dictionary must be almost orthogonal to one another). The algorithm is provably correct in that if the input signal consists of a linear combination of exactly $m$ vectors from the dictionary, the algorithm finds those $m$ vectors exactly. In addition, if the signal is not an exact combination of $m$ vectors but it does have an optimal approximation using $m$ vectors, then the algorithm returns an $m$-term linear combination whose approximation error to the input signal is within a constant factor of the optimal approximation error. If we seek $m$ vectors in our representation, the running time of OMP is $O(m d N)$. Dictionaries which are unions of orthonormal bases (which meet the geometric condition for the correctness of OMP), are of size $d=k N$, so the running time for OMP with such dictionaries is $O\left(m k N^{2}\right)$.

\section{Algorithm 4.1 (OMP) \\ INPUT: \\ - $A d \times N$ matrix $\boldsymbol{\Phi}$ \\ - A vector $v$ of measurements of length $N$ \\ - The desired number of terms $m$ in the compressed sig- nal}

OUTPUT:

- A set of $m$ indices $\lambda_{1}, \ldots, \lambda_{m}$

- An $N$-dimensional residual $\boldsymbol{r}_{m}$

PROCEDURE:

1. Initialize the residual $\boldsymbol{r}_{0}=\boldsymbol{v}$ and the iteration counter $t=1$.
2. Find the index $\lambda_{t}$ of the vector with the largest dot product with the current residual

$$
\lambda_{t}=\operatorname{argmax}_{j}\left|\left\langle\boldsymbol{r}_{t-1}, \boldsymbol{\varphi}_{j}\right\rangle\right|
$$

3. Let $\boldsymbol{P}_{t}$ be the orthogonal projection onto the span of the current vectors $\operatorname{span}\left\{\boldsymbol{\varphi}_{\lambda}: \lambda_{1}, \ldots, \lambda_{t}\right\}$. Calculate the new residual:

$$
\boldsymbol{r}_{t}=\boldsymbol{v}-\boldsymbol{P}_{t} \boldsymbol{v}
$$

\section{Increment $t$, and return to Step 2 if $t<m$.}

Note that if we had a single orthonormal basis as the dictionary $\mathcal{D}$, the representation obtained using Algorithm 4.1 is exactly the same as the projection onto the orthonormal basis. For example, if we just had a Fourier basis, the coefficients obtained from a regular Fourier transform would exactly match the coefficients obtained from the matching pursuit procedure.

\section{Data Description}

The primary data set we have used for evaluating our methods consists of traffic aggregates collected over a 20 week period (between January and June 2004) at a large Tier-1 Internet provider's IP backbone network. The dataset consists of traffic aggregates in terms of flow, packet, and byte counts. The dimensions of interest over which the aggregates are collected are:

- TCP Ports: Traffic to and from each of the 65535 TCP ports.

- UDP Ports: Traffic to and from each of the 65535 UDP ports.

- Aggregated Network Prefixes: Traffic to and from network prefixes aggregated at a set of predefined network prefixes.

The traffic aggregates were generated from flow records using traffic collection tools similar to Netflow [14], aggregated over multiple links in the provider's Internet backbone. In this particular data set, the traffic volume counts are reported on an hourly basis. For example, for each TCP port the data set contains the total number of flows, packets, and bytes on that port. The data set aggregates each metric (i.e., flows, packets, and bytes) for both incoming (i.e., traffic with this port was the destination port) and outgoing traffic (i.e., traffic with this port as the source port). Such per-port and per-prefix aggregates are routinely collected at many large ISPs and large enterprises for various traffic engineering and traffic analysis applications.

It is useful to note that such data sets permit interesting traffic analysis including observing trends in the traffic 
data, and detecting and diagnosing anomalies in the network data. For many types of network incidents of interest (outages, DoS and DDoS attacks, worms, viruses, etc.) the dataset has sufficient spatial granularity to diagnose anomalies. For example, the number of incoming flows into specific ports can be an indication of malicious scanning activity or worm activity, while the number of incoming flows into specific prefixes may be indicative of flash-crowds or DoS attacks targeted at that prefix.

For the following discussions, we consider the data in week long chunks, partly because a week appears to be the smallest unit within which constituent components of the signal manifest themselves, and also because a week is a convenient time unit from an operational viewpoint.

\section{Results}

In this section, we demonstrate how we can use sparse approximations to compress traffic time series data. We look at the unidimensional aggregates along each port/protocol pair and prefix as an independent univariate signal. In the following sections, unless otherwise stated, we work with the total number of incoming flows into a particular port. We observe similar results with other traffic aggregates such as the number of packets and the number of incoming bytes incoming on each port, and for aggregated counts for the number of outgoing flows, packets, bytes on each port-we do not present these results for brevity. We present the results only for the TCP and UDP ports and note that the compression results for aggregated address prefixes were similar.

Since an exhaustive discussion of each individual port would be tedious, we identify 4 categories of ports, predominantly characterized based on the applications that use these ports. For each of the categories the following discussion presents results for a few canonical examples.

1. High volume, popular application ports (e.g., HTTP, SMTP, DNS).

2. P2P ports (e.g., Kazaa, Gnutella, E-Donkey).

3. Scan target ports (e.g., Port 135, Port 139) .

4. Random low volume ports.

\subsection{Fourier Dictionary}

Our first attempt at selecting a suitable dictionary for compression was to exploit the periodic structure of traffic time series data. A well known fact, confirmed by several measurements $[9,10,15]$, is the fact that network traffic when viewed at sufficient levels of aggregation exhibits remarkably periodic properties, the strongest among them being the distinct diurnal component. It is of interest to identify these using frequency spectrum decomposition techniques (Fourier analysis). It is conceivable that the data can be compressed using a few fundamental frequencies, and the traffic is essentially a linear combination of these harmonics with some noisy stochastic component.

To understand the intuition behind using the frequency spectrum as a source of compression we show in Figure 2 the power spectrum of two specific ports for a single week. In each case the power spectrum amplitudes are normalized with respect to the maximum amplitude frequency for that signal (usually the mean or $0^{\text {th }}$ frequency component), and the $y$-axis is shown on a log-scale after normalization. We observe that the power spectrum exhibits only a few very high energy components. For example the central peak and the high energy band around it corresponds to the mean $\left(0^{t h}\right)$ frequency in the Fourier decomposition, while the slightly lesser peaks symmetric around zero, and close to it correspond to the high energy frequencies that have a wavelength corresponding to the duration of a day.

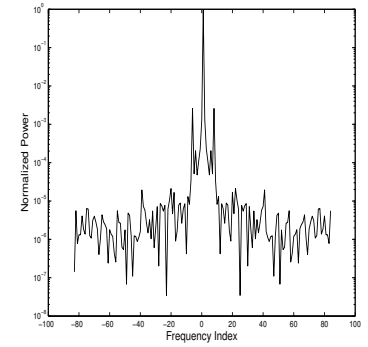

(a) Port 25/TCP

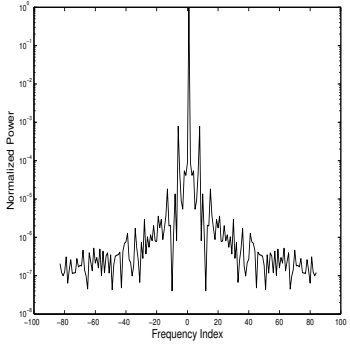

(b) Port 4662/TCP
Figure 2: Frequency power spectrum of time-series of incoming flows on specific ports over a single week

We also show the how the normalized amplitude decreases when we sort the frequency components in descending order of their amplitudes in Figure 3. We observe that there is indeed a sharp drop (the figures are in log-scale on y-axis) in the energy of the frequency components after 20-30 components for the different signals considered.

We observe that a small number of components do capture a significant portion of the energy, which suggests a rather obvious compression scheme. For each week-long time series, pick the $k$ frequencies that have the highest energies in the power spectrum. Figure 4 indicates that using 40 coefficients per week (around 40/168 $=25 \%$ of the original signal size) coefficients yields a relative error of less than 0.05 for more than $90 \%$ of all ports ${ }^{3}$. A relative error of 0.05 using our relative error metric indicates that around $95 \%$ of the original signal energy was captured in the compressed form. We observe in Figure 5 that the corresponding compressibility of UDP ports is slightly worse. 


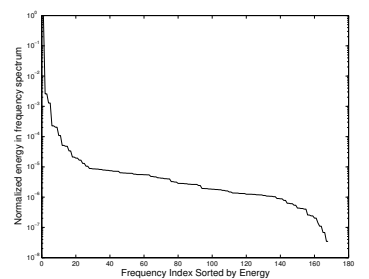

(a) Port 25/TCP

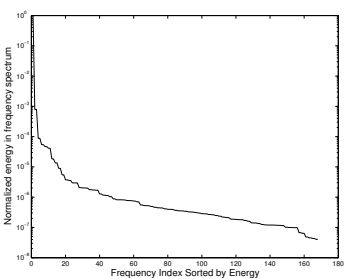

(b) Port 4662/TCP
Figure 3: Energy of the frequencies sorted in descending order for specific ports

The reason is that the traffic volumes on UDP ports tend to exhibit far lesser aggregation, in terms of absolute volumes and popularity of usage of particular ports. Intuitively one expects that with higher volumes and aggregation levels, the traffic would exhibit more periodic structure, which explains the better compression for TCP ports as opposed to UDP ports.

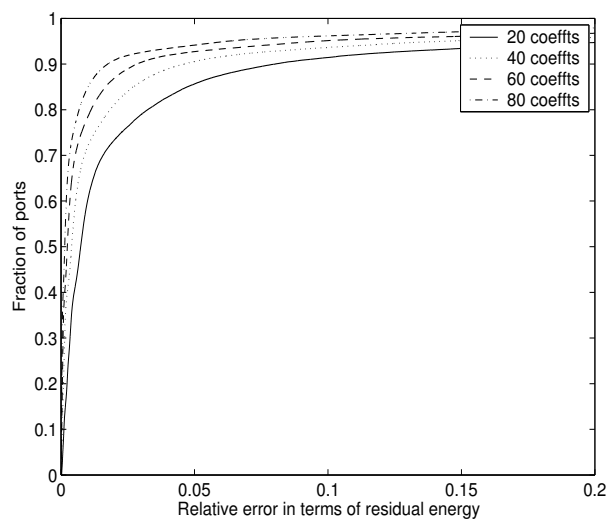

Figure 4: CDFs of relative error for TCP ports (incoming flows) with Fourier dictionary

The Fourier basis is one simple orthonormal basis. There are a host of other orthonormal bases which have been employed for compressing different datasets. Wavelets have traditionally been used for de-noising and compression in image and audio applications. The effectiveness of a wavelet basis depends on the choice of the "mother wavelet" function. However, identifying the best basis for representing either a given signal or a class of signals is a hard problem, for which only approximate answers exist using information-theoretic measures [17]. For our experiments we tried a variety of wavelet families including the well studied Daubechies family of wavelets, and other derivatives such as Symlets and Coiflets. Our observation is that the families of wavelets we tested had poorer perfor-

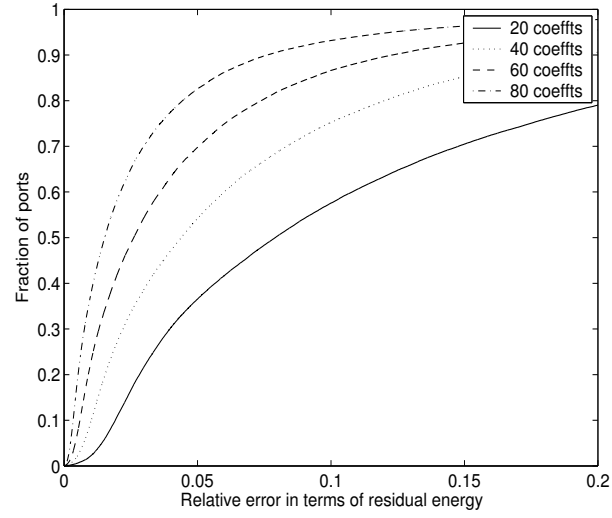

Figure 5: CDFs of relative error for UDP ports (incoming flows) with Fourier dictionary

mance when compared with the Fourier basis. Although an exhaustive discussion of choosing the ideal wavelet family is beyond the scope of this paper, our experiments with a host of wavelet families indicate that the traffic time-series cannot be efficiently compressed using wavelets (as an orthonormal basis) alone.

\subsection{Using Redundant Dictionaries}

Our choice of the Fourier dictionary was motivated by the observation that the traffic time-series when viewed at a reasonable level of aggregation possesses a significant periodic component. Therefore, using Fourier basis functions as part of the redundant dictionary seems a reasonable starting point. There are however, other interesting incidents we wish to capture in the compressed representation. Experience with traffic data indicates that interesting events with high volume (and hence high signal energy) include possibly anomalous spikes, traffic dips, and slightly prolonged high traffic incidents. Such isolated incidents, localized in time, cannot be succinctly captured using only a Fourier basis. Fortunately, these events can be modeled either using spike functions appropriately placed at different time indices, or using Haar wavelets (square waveforms) of different scales and all translations. The fully-translational Haar wavelets at all scales and all translations form a rich redundant dictionary of size $N \log N$. By contrast, the orthonormal basis of Haar wavelets is of size $N$ and consists of the Haar wavelets at all scales and only those translations which match the scale of the wavelet.

Table 1 compares a host of possible dictionaries on selected ports. Over the entire spectrum of port types, we observe that specific bases are indeed better suited than others for specific ports. For example, we observe that for some high volume and P2P ports using a Fourier dictionary gives better compression than using a wavelet or fulltranslation Haar dictionary, while for some of the random 
and scan ports, the wavelet or full-translation Haar dictionary give better compression. In some cases (e.g. port 114) we also find that using spikes in the dictionary gives the lowest compression error.

Rather than try to optimize the basis selection for each specific port, we wish to use redundant dictionaries that can best capture the different components that can be observed across the entire spectrum of ports. Hence we use redundant dictionaries composed of Fourier, fully-translational Haar, and Spike waveforms and observe that we can extract the best compression (in terms of number of coefficients selected), across an entire family of traffic time series data. We compare three possible redundant dictionaries: Fourier+ Haar wavelets (referred to as $D_{F+H}$ ), Fourier + Spikes (referred to as $D_{F+S}$ ), and Fourier + Spikes + Haar wavelets (referred to as $D_{F+H+S}$ ). Within each dictionary the error-compression tradeoff is determined by the number of coefficients chosen (Recall that a m-coefficient representation roughly corresponds to a compression ratio of $m / N)$. A fundamental property of the greedy pursuit approach is that with every iteration the residual energy decreases, and hence the error is a monotonically decreasing function of the number of modes chosen. We evaluate the error-compression tradeoffs for these different dictionaries in Figures 6 and 7, where we assume that we are constrained to use 30 coefficients (roughly corresponding to using only one-sixth of the data points for each week). We observe two main properties of using the redundant dictionary approach. First, the compressibility is substantially enhanced by expanding the dictionary to include either spikes or Haar wavelets, in addition to the periodic Fourier components, i.e., using redundant dictionaries yields better fidelity for the same storage cost as compared to a single orthonormal basis. The second property we observe with the particular choice of basis functions on the traffic data is a monotonicity property - adding a richer basis set to the dictionary helps the compressibility. For example the error-compression tradeoff that results with $D_{F+H+S}$ is never worse than either $D_{F+H}$ or $D_{F+S}$. The compression does come at a slightly higher computation cost, since the time to compress the time series depends on the size of the dictionary used, as the compression time scales in linearly with the number of vectors in the dictionary (refer Section 4).

In Figures 8 and 9 we show how the 95th percentile of the relative error across all the ports decreases as a function of the number of coefficients used for representing the traffic data for each port for TCP and UDP ports respectively. We find that after 30-35 coefficients we gain little by adding additional coefficients, i.e., the marginal improvement in the fidelity of the representation becomes less significant. We will address this issue again in Section 8 , by considering the rate of decrease of the residual as a function of the number of modes selected for specific ports, to derive

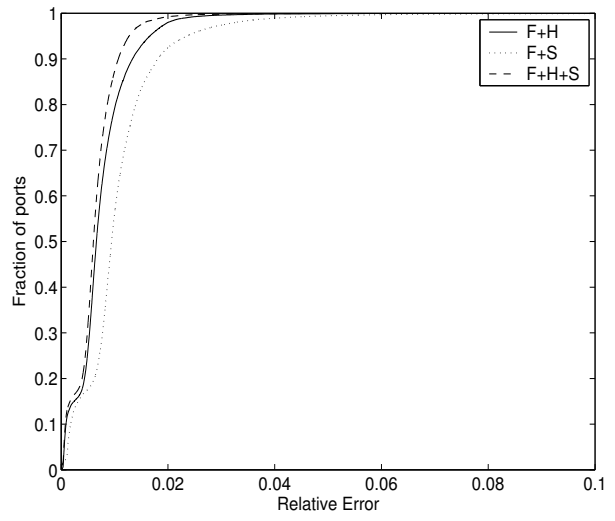

Figure 6: CDFs of relative error for TCP ports (incoming flows) with 30 coefficients for different dictionaries

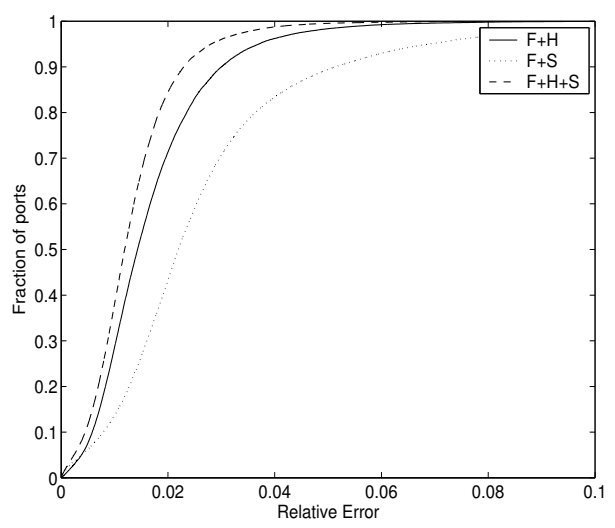

Figure 7: CDFs of relative error for UDP ports (incoming flows) with 30 coefficients for different dictionaries

stopping criteria for obtaining compressed representations.

\subsection{Analysis of Selected Modes}

We proceed to analyze the set of dictionary components that are chosen in the compressed representation using the redundant dictionaries for different ports, along different spatial and temporal dimensions. First, we are interested to see if there is substantial similarity in the set of dictionary components selected in the compressed representation across different ports. Second, we want to observe the temporal properties of compression; i.e., for a fixed traffic dimension, how does one week differ from another in terms of the components selected from the redundant dictionary? Third, we want to identify possible sources of correlation across the different traffic aggregates (flows, packets, bytes, both to and from) on a particular port of interest. Such analysis not only helps us to understand the nature of the underlying constituent components that make up each traffic 
Table 1: Compression error with 30 coefficient representation for selected TCP ports (Legend: $\mathrm{F}=$ Fourier, $\mathrm{W}=$ Orthonormal $d b 4$ wavelets, $\mathrm{H}=$ Fully-translational Haar wavelets, $\mathrm{S}=$ Spikes)

\begin{tabular}{|c|c|c|c|c|c|c|c|c|c|}
\hline \multirow{2}{*}{ Port Type } & \multirow{2}{*}{ Port Number } & \multicolumn{7}{|c|}{ Relative error with different dictionaries } \\
\cline { 2 - 9 } & & $D_{F}$ & $D_{W}$ & $D_{S}$ & $D_{H}$ & $D_{F+S}$ & $D_{F+H}$ & $D_{F+H+S}$ & $D_{H+S}$ \\
\hline \multirow{2}{*}{ High Volume } & 25 & 0.0005 & 0.0026 & 0.8446 & 0.0007 & 0.0004 & 0.0004 & 0.0004 & 0.0007 \\
\cline { 2 - 9 } & 80 & 0.0052 & 0.0256 & 0.7704 & 0.0074 & 0.0052 & 0.0018 & 0.0018 & 0.0073 \\
\hline \multirow{2}{*}{ P2P } & 1214 & 0.0003 & 0.0036 & 0.0007 & 0.8410 & 0.0003 & 0.0001 & 0.0001 & 0.0007 \\
\cline { 2 - 9 } & 6346 & 0.0009 & 0.0056 & 0.8193 & 0.0013 & 0.0009 & 0.0005 & 0.0005 & 0.0013 \\
\hline \multirow{2}{*}{ Scan } & 135 & 0.0016 & 0.0216 & 0.7746 & 0.0049 & 0.0015 & 0.0008 & 0.0008 & 0.0049 \\
\cline { 2 - 9 } & 9898 & 0.0066 & 0.0143 & 0.7800 & 0.0036 & 0.0063 & 0.0032 & 0.0032 & 0.0036 \\
\hline \multirow{2}{*}{ Random } & 5190 & 0.0023 & 0.0280 & 0.7916 & 0.0040 & 0.0023 & 0.0010 & 0.0010 & 0.0039 \\
\cline { 2 - 9 } & 114 & 0.5517 & 0.1704 & 0.0428 & 0.0218 & 0.0097 & 0.0218 & 0.0068 & 0.0068 \\
\hline
\end{tabular}

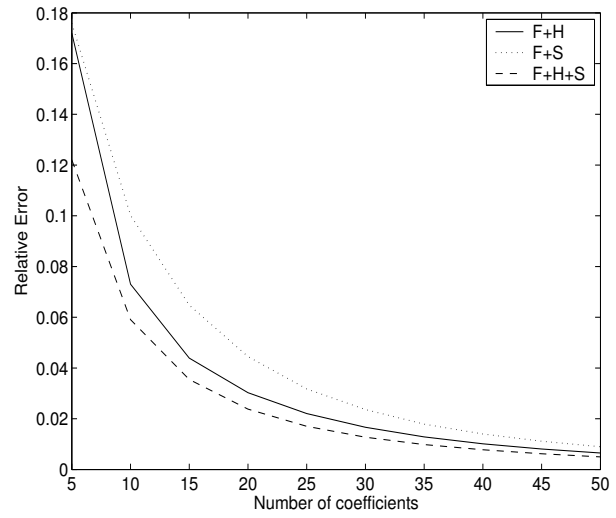

Figure 8: 95th percentile of relative error vs. number of coefficients selected for TCP ports (incoming flows)

time series but also enables us to identify possible sources of joint compression, to further reduce the storage requirements. For the discussion presented in this section, we use the dictionary $D_{F+S}$ (Fourier + Spike) as the redundant dictionary for our analysis.

\subsubsection{Spatial Analysis Across Ports}

We observe that the majority of selected dictionary components are restricted to a small number of ports-this is expected as these modes capture the minor variations across different ports, and also represent traffic spikes that may be isolated incidents specific to each port. We also observe that there are a few components that are consistently selected across almost all the ports. These components that are present across all the ports under consideration include the mean (zero-th Fourier component), the diurnal/off-diurnal periodic components, and a few other periodic components which were found to be the highest energy components in the Fourier analysis presented in Section 6.1.

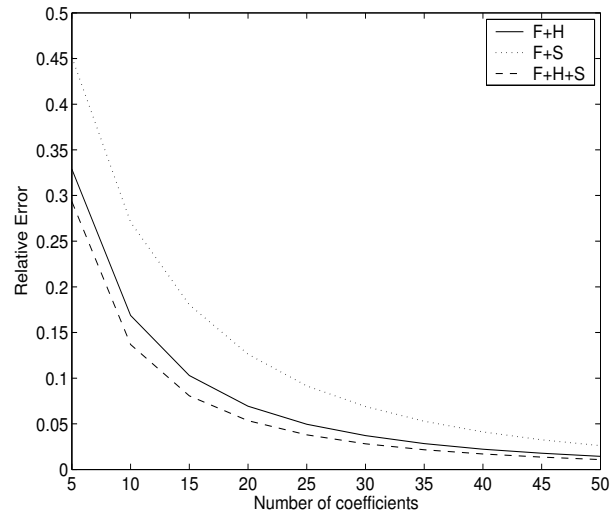

Figure 9: 95th percentile of relative error vs. number of coefficients selected for UDP ports (incoming flows)

\subsubsection{Temporal Analysis Across Multiple Weeks}

We also analyze, for specific instances of ports as defined by our four categories, the temporal stability of the set of components that are selected across different weeks over the 20 week data set, using 30 modes per week. As before, we use $D_{F+S}$ as the redundant dictionary for compression. For each dictionary component (periodic component or spike) that is selected in the compressed representation over the 20 week period, we count the number of weeks in which it is selected. We show in Figure 10 the number of components that have an occurrence count more than $x$, as a function of $x$. We observe that the majority of the components are selected only for 1-2 weeks, which indicates that these captured subtle traffic variations from week to week. To further understand the stability of the components, we divide them into 3 categories: components that occur every week, components that occurred greater than $50 \%$ of the time (i.e, were selected 10-20 times over the 20 week period), and components that occurred fewer than $50 \%$ of the time (i.e., fewer than 10 times). Table 2 presents the break- 


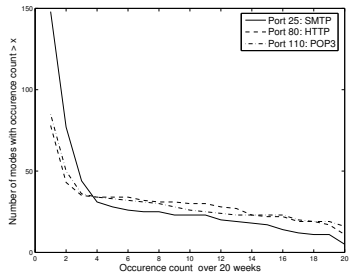

(a) High Volume Ports

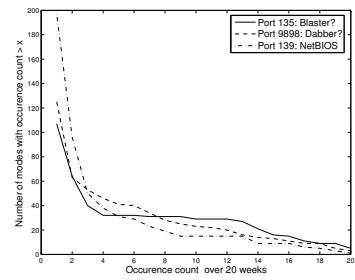

(b) Scan Target Ports

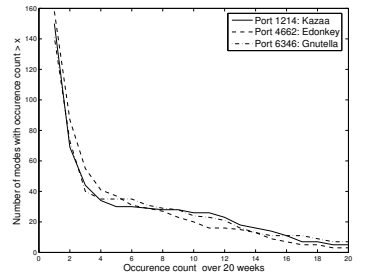

(c) P2P Ports

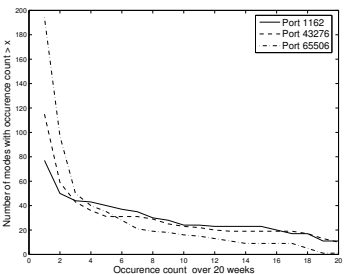

(d) Random Ports

Figure 10: Occurrence counts using a 30 coefficient representation with $D_{F+S}$ :Fourier+Spike over a 20 week period

down for the above classification for different ports in each category, and also shows the type of components that occur within each count-class. We find that across all the ports, the dictionary components that are always selected in the compressed representation correspond to periodic components such as the diurnal and off-diurnal frequencies.

The stability of a component depends not only on the fact that it was selected in the compressed representation, but also on the amplitude of the component in the compressed representation. Hence, we also analyze the amplitudes of the frequently occurring components (that occur greater than $50 \%$ of the time) across the 20 week dataset. Figures 11 and 12 show the mean and deviation of the amplitudes returned by the greedy pursuit procedure for these frequently occurring components. For clarity, we show the amplitudes of the real and imaginary part of the Fourier (periodic) components separately. For each port, we first sort the components according to the average magnitude (i.e, the energy represented by both the real and imaginary parts put together) over the 20 week period. We normalize the values of the average amplitude in both real and imaginary parts, and the deviations by the magnitude of the mean (or zero-th Fourier) component. We observe that the amplitudes are fairly stable for many Fourier components across the different port types. These results suggest that these stable (Fourier) frequencies may indeed form fundamental components of the particular traffic time series. The relative stability of amplitudes in the compressed representation also indicates that it may be feasible to build traffic models, that capture the fundamental variations in traffic, using the compressed representations.

\subsubsection{Spatial Analysis Across Traffic Metrics}

The last component of our analysis explores the similarity in the traffic data across different aggregates for a given port, within each week. One naturally expects a strong correlation between the number of flows, the number of packets, and the number of bytes for the same port, and also reasonable correlation between the total incoming volume

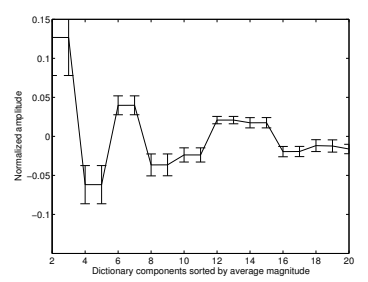

(a) Real part

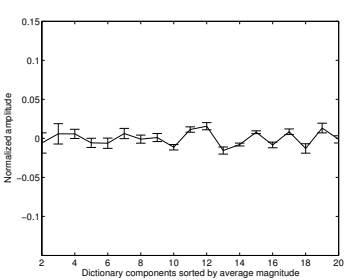

(b) Imaginary part
Figure 11: Stability of amplitudes of dictionary components selected - High volume: Port 80

and the total outgoing volume of traffic on the same port ${ }^{4}$. Figure 13 confirms this natural intuition about the nature of the traffic aggregates. We observe that for the high volume and $\mathrm{P} 2 \mathrm{P}$ application ports, more than two-thirds of the dictionary components are commonly selected across all the different traffic aggregates and we also find that more than 30 components are selected across at least 4 of the traffic aggregates (bytes, packets, flows both to and from the port). We found that such similarity in the selected components across the different aggregates is less pronounced for the scan target ports and the random ports under consideration. Our hypothesis is that the distribution of packets per flow and bytes per packet are far more regular for the high volume applications (for example most HTTP, P2P packets use the maximum packet size to get maximum throughput) than on the lesser known ports (which may be primarily used as source ports in small sized requests).

\section{Applications}

\subsection{Visualization}

One of the primary objectives of compression is to present to the network operator a high fidelity approximation that 
Table 2: Analyzing stable dictionary components for different classes of ports

\begin{tabular}{|c|c|c|c|c|c|c|c|}
\hline \multirow{2}{*}{ Port Type } & \multirow{2}{*}{ Port Number } & \multicolumn{2}{|c|}{ All 20 weeks } & \multicolumn{2}{c|}{$10-20$ weeks } & \multicolumn{2}{c|}{$0-10$ weeks } \\
\cline { 3 - 8 } & & Periodic & Spike & Periodic & Spike & Periodic & Spike \\
\hline \multirow{2}{*}{ High Volume } & 25 & 5 & 0 & 18 & 0 & 23 & 102 \\
\cline { 2 - 8 } & 80 & 11 & 0 & 19 & 0 & 15 & 33 \\
\hline \multirow{2}{*}{ P2P } & 1214 & 5 & 0 & 21 & 0 & 20 & 104 \\
\cline { 2 - 8 } & 6346 & 7 & 0 & 17 & 0 & 23 & 94 \\
\hline \multirow{2}{*}{ Scan } & 135 & 5 & 0 & 24 & 0 & 15 & 63 \\
\cline { 2 - 8 } & 9898 & 3 & 0 & 20 & 0 & 35 & 67 \\
\hline \multirow{2}{*}{ Random } & 5190 & 11 & 0 & 10 & 0 & 27 & 73 \\
\cline { 2 - 8 } & 65506 & 1 & 0 & 15 & 0 & 31 & 147 \\
\hline
\end{tabular}

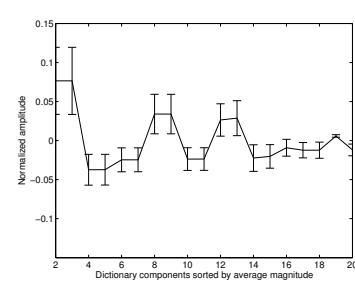

(a) Real part

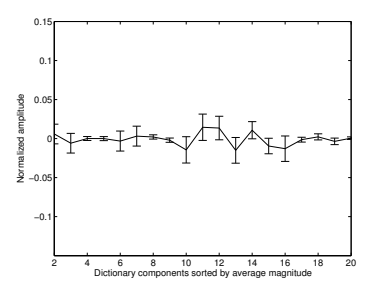

(b) Imaginary part
Figure 12: Stability of amplitudes of dictionary components selected - P2P Port: 1214

captures salient features of the original traffic metric of interest. Visualizing historical traffic patterns is a crucial aspect of traffic monitoring that expedites anomaly detection and anomaly diagnosis involving a network operator, who can use historical data as visual aids. It is therefore imperative to capture not only the periodic trends in the traffic, but also the isolated incidents of interest (for example, a postlunch peak in Port 80 traffic, the odd spike in file sharing applications, etc).

Figure 14 shows some canonical examples from each of the four categories of ports we described earlier. In each case we show the original traffic time series over a week and the time series reconstructed from the compressed representation using 1:6 compression with $D_{F+H+S}$ (Fourier + Haar + Spike). We also show the residual signal, which is the point-wise difference between the original signal and the compressed reconstruction. The traffic values are normalized with respect to the maximum traffic on that port observed for the week. We find that the compressed representations provide a high fidelity visualization of the original traffic data. Not surprisingly, the ports which exhibit the greatest amount of regularity in the traffic appear to be most easily compressible and the difference between the actual and compressed representation is almost negligible

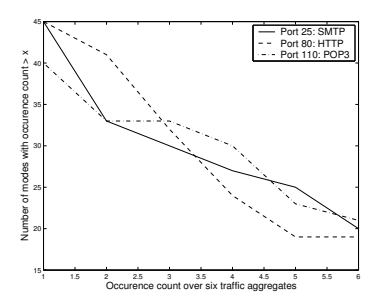

(a) High Volume Ports

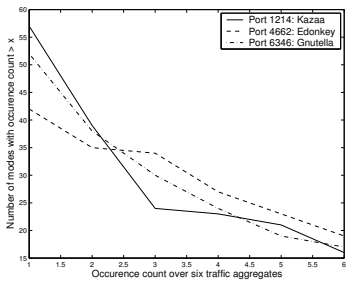

(b) P2P Ports
Figure 13: Occurrence counts using 30 coefficient representation with $D_{F+S}$ :Fourier+Spike over different traffic aggregates for a single week

for these cases. It is also interesting to observe in each case that the compressed representation captures not only the periodic component of the signal, but also traffic spikes and other traffic variations.

\subsection{Traffic Trend Analysis}

Analyzing trends in traffic is a routine aspect in network operations. Operators would like to understand changes and trends in the application mix that is flowing through the network (e.g. detecting a a new popular file sharing protocol). Understanding traffic trends is also crucial for traffic engineering, provisioning, and accounting applications. It is therefore desirable that such trend analysis performed on the compressed data yields accurate results when compared to similar trend analysis on the raw (uncompressed) data. A simple method to extract trends over long timescales is to take the weekly average, and find a linear fit (using simple linear regression to find the slope of the line of best fit) to the weekly averages over multiple weeks of data. In Figure 15 , we plot the relative error in estimating such a linear trend. We estimate the trend using 20 weeks of data for dif- 


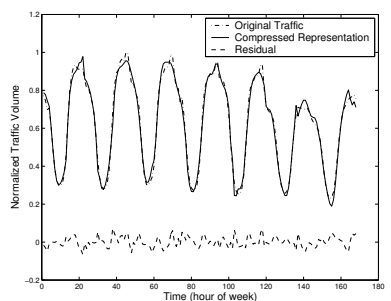

(a) Port 80

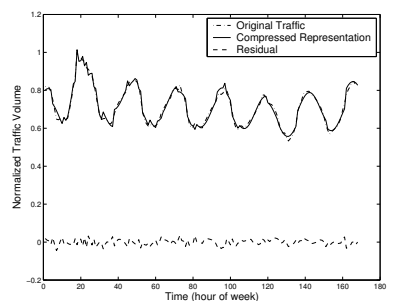

(b) Port 6346

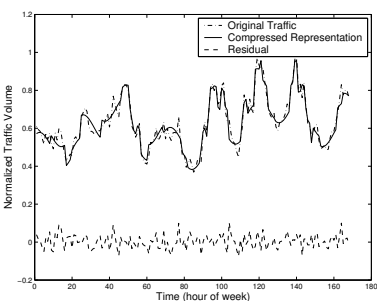

(c) Port 9898

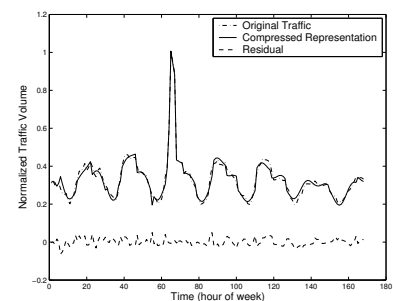

(d) Port 43726

Figure 14: Miscellaneous Ports using $D_{F+H+S}$ : Fourier + Haar Wavelets + Spikes

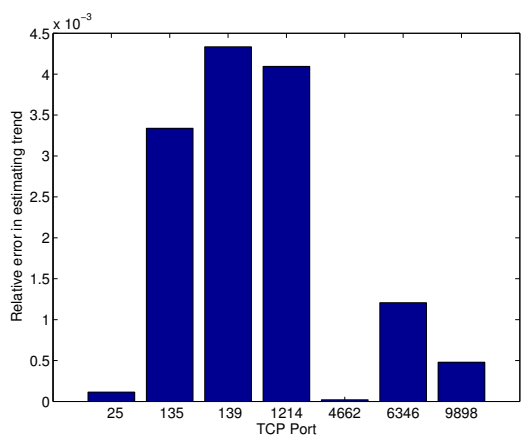

Figure 15: Relative error in estimating traffic trends

ferent ports, and in each case we estimate the slope of the best linear fit on the raw data and on the compressed data (using a 30 coefficient representation using $D_{F+H+S}$ ). We observe that across the different ports, the relative error in estimating the trend is less than $0.5 \%$, which reaffirms the high fidelity of the compression techniques.

\subsection{Modeling and Anomaly Detection}

We observed in Section 6.3 that the underlying fundamental components are stable (both in terms of occurrence and their amplitudes) over time. It is conceivable that traffic models for anomaly detection can be learned on the compressed data alone. Our initial results suggest that traffic models [15] learned from compressed data have almost identical performance to models learned from uncompressed data, and hence compression does not affect the fidelity of traffic modeling techniques. Ongoing work includes evaluating different models for building prediction models for real-time anomaly detection using accurate yet parsimonious prediction models generated from the insights gained from the compression procedures.

\section{Discussion}

\subsection{Stopping Criteria}

In our experiments, we fixed the number of coefficients across all ports. One can imagine a host of stopping criteria to apply. One particularly interesting observation is that in many of the cases, a few of which are depicted in Figure 16, we find that the residual energy has a distinct knee beyond which the rate of drop in the residual energy is significantly lower. Intuitively one can imagine as the knee corresponding to the stochastic noise component of the original signal, which cannot be efficiently represented using any fundamental component. Note that the anomalous incidents such as spikes or glitches are usually captured before we hit the knee of the curve, as observed in Section 7.1. This raises the possibility that we have a robust representation of the original signal-one that does not change with the addition of noise as there are diminishing returns for any added effort aimed at modeling the noise component, which are not necessarily of interest either from a visualization or modeling perspective. We have

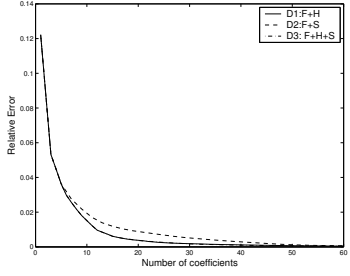

(a) Port 80

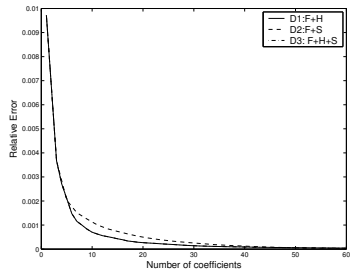

(b) Port 1214
Figure 16: Evaluating Stopping Criteria: Relative Error vs. number of coefficients

performed independent experiments with synthetic time series signals, similar to traffic time series (sinusoidal signals, 
with spikes and different noise patterns thrown in). We observe that in almost all the cases we observe a distinct knee in the redundant dictionary decomposition, once the fundamental high energy components get picked. We also find that the asymptotic slope of the curve of the residual energy beyond the knee has a unique signature that is characterized by the nature of the noise component (Gaussian or "White" vs. Power-law or "Colored"), and the redundant dictionary used.

\subsection{Smaller Scales}

At an appropriate aggregation level, network traffic will exhibit some periodicities. Traffic time series data from a variety of settings (enterprise and university) also confirm this hypothesis. These data typically represent the aggregate traffic at the border of a reasonably large network with fairly high aggregation levels. We believe that the methods for time-series compression using matching pursuit with redundant dictionaries are still applicable to data even at slightly lower scales of aggregation.

One of the objectives of compressing the time series is to enable different scales of time resolution for anomaly detection. It is imperative that the time scale for detecting traffic anomalies be less than the minimum time required for a large network attack to saturate. When the compression is applied to traffic aggregates at finer time granularities (e.g. for each week if we had volume counts for each five minute bin instead of hourly time bins), one expects that the effective compression would be better. The rationale behind the intuition arises from the fact that the high energy fundamental components correspond to relatively low frequency components, and such pronounced periodicities are unlikely to occur at finer time-scales. As a preliminary confirmation of this intuition, we performed the same compression procedures on a different data set, consisting of 5 minute traffic rates collected from SNMP data from a single link. Note that with 5-minute time intervals, we have $168 \times 12=2016$ data points per week. Figure 17 the relative error as a function of the number of coefficients used in the compressed representation (using $D_{F+S}$ ). We observe that with less than 40 ( $=2 \%$ of the original space requirement) coefficients we are able to adequately compress the original time-series (with a relative error of less than 0.005), which represents significantly greater possible compression than those observed with the hourly aggregates.

\subsection{Encoding Techniques}

We observed that with larger dictionaries that include fulltranslation wavelets, we can achieve better compression. There is, however, a hidden cost in the effective compression with larger dictionaries as the indices of a larger dic-

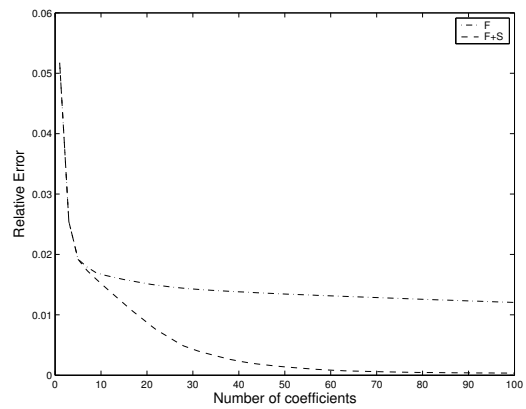

Figure 17: Compressing SNMP data collected at five minute intervals from a single link

tionary potentially require more bits to represent than the indices of a smaller dictionary. One can imagine better ways of encoding the dictionary indices (e.g., using Huffman coding) to reduce the amount of space used up for storing the dictionary indices in addition to the component amplitudes. Our work explored the potential benefit of using signal processing methods for lossy compression and we observed that there is a substantial reduction in the storage requirement using just the methods presented in this paper. Many compression algorithms use lossy compression techniques along with efficient encoding techniques (lossless compression) to get the maximum compression gain, and such combinations of lossy and lossless compression methods can be explored further.

\subsection{Joint Compression}

We observe that there are multiple sources of correlation across the different traffic dimensions that may be additionally utilized to achieve better compression. The temporal stability of the compressed representations (Section 6.3.2) suggests there is scope for exploiting the similarity across different weeks for the same traffic aggregate. For example, we could build a stable model over $k$ weeks of data for each port/prefix and only apply the sparse approximation to the difference of each particular week from the model. Alternately one could imagine applying the simultaneous compression algorithms [16] across the different weeks for the same port. The simultaneous compression algorithms approximate all these signals at once using different linear combinations of the same elementary signals, while balancing the error in approximating the data against the total number of elementary signals that are used. We also observed that there is reasonable correlation in spatial dimensions, since the compressed representation of different traffic aggregates such as flows, packets, and bytes show significant similarity (Section 6.3.3).

The observations of the low dimensionality of network traffic data across different links also raises the possibility of using Principal Component Analysis (PCA) [10] for 
extracting better spatial compression, both across different traffic aggregates (e.g. different ports, across time) and across different measurements (e.g. across per-link, perrouter counts). PCA like methods can be used to extract the sources of correlation before one applies redundant dictionary approaches to compress the traffic data. For example we can collapse the 20 week data set for a single port into a single matrix of traffic data, on which PCA like techniques can be applied to extract the first few common components, and the redundant dictionary can be applied on the residual (the projection on the non-principal subspace) to obtain a higher fidelity representation.

\section{Conclusions}

There is a pressing need for fine-grained traffic analysis at different scales and resolutions across space and time for network monitoring applications. Enabling such analysis requires the ability to store large volumes of historical data across different links, routers, and customers, for generating visual and diagnostic aids for network operators. In this paper, we presented a greedy pursuit approach over redundant dictionaries for compressing traffic time series data, and evaluated them using measurements from a large ISP. Our observations indicate that the compression models present a high fidelity representation for a wide variety of traffic monitoring applications, using less than $20 \%$ of the original space requirement. We also observe that most traffic signals can be compressed and characterized in terms of a few stable frequency components. Our results augur well for the visualization and modeling requirements for large scale traffic monitoring. Ongoing work includes evaluating and extracting sources of compression across other spatial and temporal dimensions, and evaluating the goodness of traffic models generated from compressed representations.

\section{References}

[1] Barford, P., Kline, J., Plonka, D., And Ron, A. A Signal Analysis of Network Traffic Anomalies. In Proc. of ACM/USENIX Internet Measurement Workshop (2002).

[2] Duffield, N. G., Lund, C., And Thorup, M. Charging From Sampled Network Usage. In Proc. of ACM SIGCOMM Internet Measurement Workshop (2001).

[3] Estan, C., Savage, S., and Varghese, G. Automatically Inferring Patterns of Resource Consumption in Network Traffic. In Proc. of ACM SIGCOMM (2003).

[4] Frossard, P., Vandergheynst, P., i Ventura, R. M. F., And KUNT, M. A posteriori quantization of progressive matching pursuit streams. IEEE Trans. Signal Processing (2004), 525-535.

[5] Gilbert, A. C., Muthukrishnan, S., and Strauss, M. J. Approximation of functions over redundant dictionaries using coherence. In Proc. of 14th Annual ACM-SIAM Symposium on Discrete Algorithms (2003).

[6] GRIBOnVAL, R., AND BACRY, E. Harmonic decomposition of audio signals with matching pursuit. IEEE Trans. Signal Processing (2003), 101-111.
[7] INDYK, P. High-dimensional computational geometry. PhD thesis, Stanford University, 2000.

[8] Krishnamurthy, B., Sen, S., Zhang, Y., and Chen, Y. Sketch-based Change Detection: Methods, Evaluation, and Applications. In Proc. of ACM/USEINX Internet Measurement Conference (2003).

[9] Lakhina, A., Crovella, M., and Diot, C. Diagnosing network-wide traffic anomalies. In Proc. of ACM SIGCOMM (2004).

[10] Lakhina, A., Papagiannaki, K., Crovella, M., Diot, C., KOLACZYK, E., AND TAFT, N. Structural analysis of network traffic flows. In Proc. of ACM SIGMETRICS (2004).

[11] LEMPEL, A., AND ZIV, J. Compression of individual sequences via variable-rate coding. IEEE Transactions on Information Theory 24 , 5 (1978), 530-536.

[12] Mallat, S., And Zhang, Z. Matching pursuits with time frequency dictionaries. IEEE Trans. Signal Processing 41, 12 (1993), 3397-3415.

[13] Miller, A. J. Subset selection in regression, 2nd ed. Chapman and Hall, London, 2002.

[14] Cisco Netflow. http://www.cisco.com/warp/public/ $732 / \mathrm{Tech} / \mathrm{nmp} / \mathrm{net}$ low/index.shtml.

[15] Roughan, M., Greenberg, A., Kalmanek, C., Rumsewicz, M., YATES, J., AND ZHANG, Y. Experience in measuring internet backbone traffic variability: Models, metrics, measurements and meaning. In Proc. of International Teletraffic Congress (ITC) (2003).

[16] Tropp, J. A., Gilbert, A. C., And Strauss, M. J. Algorithms for simultaneous sparse approximation part i: Greedy pursuit. $s u b-$ mitted (2004).

[17] Zhuang, Y., AND BARAS, J. S. Optimal wavelet basis selection for signal representation. Tech. Rep. CSHCN TR 1994-7, Institute for Systems Research, Univ. of Maryland, 1994.

\section{Notes}

${ }^{1}$ Typically, $k^{\prime}$ is less than $k$, i.e. the magnitudes of the amplitudes of the dictionary components are less than the original time series.

${ }^{2}$ The slowest step in OMP is choosing the waveform which maximizes the dot product with the residual at each step. We can speed up this step with a Nearest Neighbors data structure [7] and reduce the time complexity for each iteration to $N+\operatorname{polylog}(d)$.

${ }^{3}$ Note that for each Fourier coefficient, we need to store both the real part and the imaginary part. It appears that we may actually need twice the space. However, the amplitudes for frequency $f$ and frequency $-f$ are the same (except that they are complex conjugates of one another), we can treat them as contributing only two coefficients to the compressed representation together in total as opposed to four coefficients.

${ }^{4}$ We however note that there may be certain exceptional situations (e.g., worm or DDoS attacks that use substantially different packet and byte types) where such stable correlations between the flow, packet, and byte counts may not always hold. 